\title{
Use of Robotic Technology to Assess Brain Function
}

\author{
Stephen H. Scott \\ Department of Biomedical and Molecular Sciences, \\ Queen's University \\ Kingston, Canada \\ steve.scott@queensu.ca
}

\section{Extended Abstract}

Clinical assessment plays a crucial role in all facets of patient care, from diagnosing the specific disease or injury, to management and monitoring of rehabilitation strategies to ameliorate dysfunction. Most assessment scales for sensorimotor function are subjective in nature with relatively coarse rating systems, reflecting that it is difficult for even experienced observers to discriminate consistently small changes in performance using only the naked eye. Our general hypothesis is that robotic technologies can provide an objective approach to quantify sensory, motor and cognitive impairments. We developed a robotic device called KINARM, an exoskeleton robot that is attached to the arm and permits movements at the shoulder and elbow joints in the horizontal plane [1]. The system includes two KINARM robots to assess each arm, and an integrated virtual reality system that projects objects onto the horizontal workspace.

We have developed a suite of behavioural tasks to quantify sensory, motor and cognitive impairments in subjects with neurological disorders. I will describe a number of tasks to assess various aspects of motor function, including a visual guided reaching task to quantify goal-directed motor actions [2], a bimanual task to assess the ability to coordinate movements of the two arms [3], a posture perturbation task to assess use of limb afferent feedback for motor action [4], an object hit task to assess rapid motor skills [5], and an object hit and avoid task to quantify rapid motor and cognitive processing [6]. Subject performance was characterized using a set of parameters related to the spatiotemporal features of each motor action. A large cohort of healthy control subjects $(n>100)$ completed each task in order to develop normative models on healthy performance considering factors such as age, sex and handedness. Impairments were defined as performance outside the 95\% range observed for healthy controls. These studies highlight a broad range of impairments that can occur following stroke. First, the unaffected limb often displays impairments in motor function particularly for subjects with stroke in the right hemisphere. Second, the ability to make rapid motor decisions and motor actions are commonly impaired, but are not captured with existing clinical tools. Third, there is substantial heterogeneity in impairments across individuals with stroke, suggesting the need for patient-specific rehabilitation programs.

We have also developed other robotic platforms for clinical assessment including end-point robots that can be grasped by the subjects, and systems that can be used while standing to assess coordination between voluntary control of the arms and whole-body balance control. Eye-tracking technologies can also be integrated into the systems to quantify oculomotor function and eye-hand coordination. These platforms are being used to quantify impairments in many disorders/injuries beyond stroke, including ALS, Parkinson's disease, spinal cord injury, transient ischemic attack, concussion, cardiac arrest, and pre- and post-operative assessments for a range of neurological and non-neurological interventions.

\section{References}

[1] S. H. Scott, "Apparatus for measuring and perturbing shoulder and elbow joint positions and torques during reaching," J. Neurosci. Methods, vol. 89, pp. 119-127, 1999.

[2] A. M. Coderre, A. Abou Zeid, S. P. Dukelow, M. J. Demmer, K. D. Moore, M. J. Demers, H. Bretzke, T. M. Herter, J. I. Glasgow, K. E. Norman, S. D. Bagg, and S. H. Scott, "Assessment of upper-limb sensorimotor function of subacute stroke subjects using visually-guided reaching," J. of Neurorehabilitation and Neural Repair, vol. 24, pp. 528-541, 2010.

[3] C. R. Lowrey, C. P. T. Jackson, S. D. Bagg, S. P. Dukelow, and S. H. Scott, "A novel robotic task for assessing impairments in bimanual coordination post-stroke," Inter. J. Physical Med. Rehab., 2014. 
[4] T. C. Bourke, A. M., Coderre, S. D. Bagg, S. P. Dukelow, K. E. Norman, and S. H. Scott, "Corrective responses to a postural perturbation of the upper limb are impaired bilaterally in people with stroke," J. Neuroeng. Rehab., vol. 12, pp. 7, 2015.

[5] K. Tyryshkin, A. M. Coderre, J. I. Glasgow, T. M. Herter, S. P. Dukelow, S. D. Bagg, and S. H. Scott, "A robotic hitting task to quantify sensorimotor impairments in subjects with stroke," J. Neuroeng. Rehab., vol. 11, pp. 47, 2014.

[6] T. C. Bourke, C. R. Lowrey, S. P. Dukelow, S. D. Bagg, K. E. Norman, and S. H. Scott, "A robot-based behavioural task to quantify impairments in rapid motor decisions and actions after stroke," J. Neuroeng. Rehab., 2015 (submitted). 